\title{
Utility of C-peptide for a reliable estimate of insulin secretion in children with growth hormone deficiency
}

\author{
Alessandro Ciresi, Floriana Cicciò, Stefano Radellini, Carla Giordano*

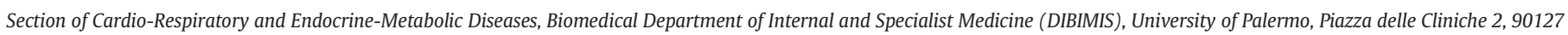
Palermo, Italy

\section{A R T I C L E I N F O}

\section{Article history:}

Received 5 November 2015

Received in revised form 2 May 2016

Accepted 7 May 2016

Available online 09 May 2016

\section{Keywords:}

Growth hormone treatment

Glucose metabolism

Insulin secretion

C-peptide

\begin{abstract}
A B S T R A C T
Objective: GH treatment (GHT) can lead to glucose metabolism impairment through decreased insulin sensitivity and impaired pancreatic $\beta$-cell function, which are the two key components of the pathogenesis of diabetes. Therefore, in addition to insulin sensitivity, during GHT it is very important to perform a reliable evaluation of insulin secretion. However, conflicting data exist regarding the insulin secretion in children during GHT. C-peptide provides a more reliable estimate of $\beta$-cell function than insulin, but few studies evaluated it during GHT. Our aim was to assess the usefulness of C-peptide in the evaluation of insulin secretion in GH deficiency (GHD) children. Design: In 48 GHD children, at baseline and after 12 and 24 months of GHT, and in 56 healthy subjects we evaluated fasting and glucagon-stimulated $\left(\mathrm{AUC}_{\mathrm{Cpep}}\right) \mathrm{C}$-peptide levels in addition to other commonly used secretion indexes, such as fasting and oral glucose tolerance test-stimulated insulin levels $\left(A U C_{I N S}\right)$, Homa- $\beta$, and insulinogenic index. The main outcomes were the change in C-peptide during GHT and its correlation with the auxological and hormonal parameters.

Results: At baseline GHD children showed a significant lower $\mathrm{AUC}_{\mathrm{Cpep}}(\mathrm{p}=0.006)$, while no difference was found for the other indexes. Both fasting C-peptide (beta 0.307, $\mathrm{p}=0.016$ ) and $\mathrm{AUC}_{\text {Cpep }}$ (beta 0.379, $\mathrm{p}=0.002$ ) were independently correlated with IGF-I SDS, while no correlation was found for all other indexes. After 12 months an increase in Homa- $\beta(p<0.001)$, fasting C-peptide $(p=0.002)$ and $\operatorname{AUC}_{\text {cpep }}(p<0.001)$ was found. At multivariate analysis, only fasting $\mathrm{C}$-peptide (beta $0.783, \mathrm{p}=0.001$ ) and $\mathrm{AUC}_{\mathrm{Cpep}}$ (beta $0.880, \mathrm{p}<0.001$ ) were independently correlated with IGF-I SDS.

Conclusions: C-peptide, rather than the insulin-derived indexes, has proved to be the most useful marker of insulin secretion correlated to IGF-I levels in GHD children. Therefore, we suggest the use of glucagon test both as diagnostic test for the GH assessment and as a useful tool for the evaluation of insulin secretion during GHT in children.
\end{abstract}

(C) 2016 Elsevier Ltd. All rights reserved.

\section{Introduction}

Growth hormone $(\mathrm{GH})$ is known to play a significant metabolic role [1]. Untreated GH deficiency (GHD) in children, as well as in adults, is associated with abnormalities in body composition with increased visceral fat, dyslipidemia, increased peripheral inflammatory markers and cardiovascular risk [2-4]. In addition, GHD is often associated with impaired glucose metabolism and the increased prevalence of type 2 diabetes is likely due to the decreased insulin sensitivity and to the inadequate $\beta$-cell ability to counteract the insulin resistance $[5,6]$. Moreover, GH has an insulin antagonist effect $[7,8]$ in addition to

\footnotetext{
Abbreviations: $\mathrm{GH}$, growth hormone; GHT, growth hormone treatment; GHD, growth hormone deficiency; GST, glucagon stimulation test.

* Corresponding author.

E-mail address: carla.giordano@unipa.it (C. Giordano).
}

directly stimulate $\beta$-cell mass and insulin secretion [9-11] and these effects can induce important changes in glucose metabolism, leading to affect both insulin sensitivity and secretion [12]. In in vivo studies, a reduction in insulin sensitivity, mainly correlated with an increase in insulin levels, has been widely demonstrated in children during GH treatment (GHT) [13]. Patients who develop insulin resistance during GHT can represent a population at risk for diabetes and monitoring of glucose and insulin metabolism during GHT has been recommended $[14,15]$. Given that both the decreased insulin sensitivity and the impaired $\beta$-cell function are two key components of the pathogenesis of type 2 diabetes, it is very important to perform a reliable evaluation of insulin secretion in subjects potentially at risk of glucose metabolism impairment, as in children during GHT. In addition, a mutual relationship between insulin secretion and GH/IGF-I axis is well established in healthy subjects, as well as in diabetic patients $[16,17]$, so it is interesting to examine whether this relationship is maintained in GHD children during GHT. 
Several parameters, both fasting and stimulated, have been used to evaluate the change in insulin secretion during GHT, but with discordant results. The evaluation of $\beta$-cell function based on fasting parameters has not always proved to be a reliable measure and the existing data about the effect of GHT on insulin secretion are controversial. Indeed, both an increase [18] and a decrease in insulin secretion, in terms of inadequate adaptation of insulin secretion when the insulin sensitivity deteriorates $[19,20]$, have been demonstrated. We already showed in children during GHT a worsening of the oral disposition index, which declines before the glucose levels rise into the diabetic range [6]. Given these discrepancies, in our hypothesis a more accurate estimation of the effect of $\mathrm{GH}$ on $\beta$-cell function may come by analyzing the C-peptide secretion. It is known that $\mathrm{C}$-peptide provides a more reliable estimate of $\beta$-cell function in healthy children and it better correlates with the gold standard hyperglycemic clamp than insulin levels [21]. However, very few studies have evaluated the C-peptide levels in children during GHT $[18,22]$.

The aim of this study was to assess the reliability of both fasting and stimulated C-peptide in the evaluation of insulin secretion and its correlation with the auxological and hormonal parameters in GHD children during GHT.

\section{Materials and methods}

We prospectively evaluated 48 children ( $32 \mathrm{M}, 16 \mathrm{~F}$; mean age $9.8 \pm$ 2.1 years; range 3.7-12.9) affected by isolated idiopathic GHD consecutively admitted to the Section of Endocrinology of the University of Palermo during the years 2012-2013. Fifty-six healthy subjects, matched for sex ( $39 \mathrm{M}, 17 \mathrm{~F}$ ), age (mean age $10.2 \pm 1.3$ years; range $5.1-12$ ), stature and pubertal status, were recruited as a control group at baseline among children referred for short stature. In this group of children the screening for short stature did not reveal endocrine alterations and GHD was excluded by $\mathrm{GH}$ peak $>10 \mu \mathrm{g} / \mathrm{l}$ after two stimuli.

We excluded children affected by multiple pituitary hormone deficiency or receiving other hormonal replacement treatment. All children, even the older ones, were in the first or second stage of sexual development according to the criteria of Marshall and Tanner [23] to avoid any interference of puberty on the metabolic parameters analyzed, and they maintained the prepubertal hormonal status during the observation period (i.e. FSH and $\mathrm{LH}<1 \mathrm{mU} / \mathrm{ml}$, total testosterone and $17 \beta$-Estradiol $<0.50 \mathrm{ng} / \mathrm{ml}$ and $<5 \mathrm{pg} / \mathrm{ml}$ in males and females, respectively).

The diagnosis of GHD was established by the clinical, auxological, radiological and biochemical criteria of the GH Research Society [24]. As auxological data we considered height and growth velocity 1 year before the diagnosis. Clinical and auxological criteria included height $>2$ standard deviations (SD) below the mean and a growth velocity over 1 year $>1$ SD below the mean for age, or a decrease in height SD of $>0.5$ over 1 year or, without severe short stature, a growth velocity $>2$ SD below the mean over 1 year or, finally, height $>1.5$ SD below the midparental height.

As radiological criteria we considered a bone age delay, estimated from an x-ray of the left wrist and hand and evaluated according to methods of Greulich and Pyle, of at least 1 year with respect to the chronological age [25]. Biochemically GHD was demonstrated by the failure of GH to respond to two stimuli, with GH peaks below $10 \mu \mathrm{g} / \mathrm{l}$. Neuroimaging, with magnetic resonance of the hypothalamic-pituitary region, was performed in GHD children with more severe GHD, i.e. with GH peak $\leq 3 \mu \mathrm{g} / \mathrm{l}$ (No. 23 children). Among them, 4 patients showed a pituitary hypoplasia and 3 a partial empty sella.

All patients enrolled were treated with GH for at least 24 months. The patients received GH once daily at bedtime with a pen injection system. Insulin-like growth factor (IGF)-I levels and the growth velocity have allowed us to determine the GH dose. Specifically, the main targets were arbitrarily IGF-I levels between 0.5 and 1.5 SDS and growth velocity $>0.5$ SDS. The initial daily dose of GH was $0.025 \mathrm{mg} / \mathrm{kg}$ and it has been gradually increased by $0.002-0.003 \mathrm{mg} / \mathrm{kg}$ /day every 6 months (mean daily dose of $0.028 \mathrm{mg} / \mathrm{kg}$ from months 6 to $12 ; 0.031 \mathrm{mg} / \mathrm{kg}$ from months 12 to $18 ; 0.033 \mathrm{mg} / \mathrm{kg}$ from months 18 to 24 ).

\subsection{Study protocol}

In all children, at baseline, GH secretion was assessed by arginine and glucagon (GST) stimulation test, performed in two different days. During arginine test, blood samples were obtained at 0, 30, 60, 90, 120 min after the administration of the stimulus (arginine monohydrochloride: $0.5 \mathrm{~g} / \mathrm{kg}$ up to $30 \mathrm{~g}$. given intravenously over $30 \mathrm{~min}$ ) for GH measurements. During GST, blood samples were collected at $0,30,60,90,120,150,180$ and 240 min after the injection of $30 \mu \mathrm{g} / \mathrm{kg}$ (up to $1.000 \mu \mathrm{g}$ ) intramuscularly of glucagon (GlucaGen, NovoNordisk, Bagsvaerd, Denmark), for the measurements of both $\mathrm{GH}$ as diagnostic tool for the GHD [26,27] and C-peptide concentrations as estimate of insulin secretion. The area under the curve (AUC) of C-peptide $\left(\mathrm{AUC}_{\mathrm{Cpep}}\right)$ during GST was also calculated using the trapezoidal rule.

In a different day, a blood sample was drawn after an overnight fast for the measurement of Hemoglobin A1c (HbA1c), plasma glucose, insulin and IGF-I concentrations. This sample also served as the baseline sample for an oral glucose tolerance test (OGTT). Blood samples were collected every $30 \mathrm{~min}$ for $2 \mathrm{~h}$ for glucose and insulin measurements. AUC of glucose $\left(\mathrm{AUC}_{\mathrm{GLU}}\right)$ and insulin $\left(\mathrm{AUC}_{\mathrm{INS}}\right)$ during OGTT was calculated using the trapezoidal rule.

After the diagnosis of GHD was made, in GHD children in addition to the height, body mass index (BMI) and waist circumference (WC) measurements, we performed both OGTT and GST after 12 and 24 months of GHT. Height, BMI, WC and IGF-I were expressed as SD score (SDS) due to the wide age range of patients.

The estimation of the basal insulin secretion included fasting insulin levels and the homeostasis model assessment for $\beta$-cell function (Homa- $\beta$ ) index [28]. The early insulin-secretion rate was evaluated using the insulinogenic index [29], while the stimulated total insulin secretion was evaluated by $\mathrm{AUC}_{\mathrm{INS}}$. In addition, an estimate of the endogenous insulin secretion was obtained from C-peptide and $\mathrm{AUC}_{\mathrm{Cpep}}$.

As surrogate estimates of insulin sensitivity we considered the homeostasis model assessment estimate of insulin resistance (Homa-IR) [28], the quantitative insulin sensitivity check index (QUICKI) [30] and the insulin sensitivity index (ISI), a composite index derived from the OGTT and validated by Matsuda and DeFronzo [31]. In the control subjects, these evaluations were performed only at baseline.

The institutional Ethics Committee of the University of Palermo approved this study. At the time of hospitalization, an informed consent for the scientific use of the data was obtained from both the participants and their parents.

\subsection{Hormone and biochemical assays}

All biochemical data were collected after overnight fasting. Glucose and HbA1c were measured in the centralized accredited laboratories with standard methods. Serum insulin was measured by ELISA (DRG Instruments $\mathrm{GmbH}$, Germany). The sensitivity of the method was $1 \mathrm{IU} / \mathrm{ml}$. The normal insulin range (IU/ml) was 5-19. Serum GH levels were measured by immunoradiometric assay using commercially available kits (Radim, Italy). The sensitivity of the assay was $0.04 \mu \mathrm{g} / \mathrm{l}$. The intra and inter-assay coefficients of variation (CV) were $2.5-3.9$ and $3.8-5.0 \%$, respectively. We reported GH concentrations in $\mu \mathrm{g} / \mathrm{l}$ of IS $98 / 574$. Serum total IGF-I was assayed in the same laboratory with the ELISA method (OCTEIA IGF-I kit, IDS Inc., Fountain Hills, AZ, USA). The sensitivity of the method was $1.9 \mu \mathrm{g} / \mathrm{l}$. The inter- and intra-assay CV values were 77.1 and $2.3-3.5 \%$ respectively, at IGF-I levels of 90.7-186 and 66.7$120.9 \mu \mathrm{g} / \mathrm{l}$ respectively. The normal ranges (males and females combined) of total IGF-I levels ( $\mu \mathrm{g} / \mathrm{l})$ were: $12-108$ (0-1 years); $13-100$ (1-3 years); 26-280 (3-6 years); 85-230 (6-9 years); 98-404 (912 years); $142-525$ ( $12-15$ years); 146-415 (15-20 years). Values 
were expressed as SDS according to the normative data provided by the manufacturer. Serum C-peptide was measured by electrochemiluminescence immunoassay method (Roche Diagnostics Deutschland $\mathrm{GmbH}$ ). The sensitivity of the method was $0.01 \mathrm{ng} / \mathrm{ml}$. The normal range $(\mathrm{ng} / \mathrm{ml})$ was $0.010-40.0$.

\subsection{Statistical analysis}

The Statistical Packages for Social Sciences SPSS version 17 was used for data analysis. Baseline characteristics were presented as mean \pm SD or as median values \pm interquartile range (IR) for continuous variables, when appropriate. Normality of distribution for the quantitative variables was assessed with the Kolmogorov-Smirnov test. The differences between groups were evaluated with the $t$-test if with normal distribution or with Mann-Whitney test (non-parametric test) if without normal distribution. All differences in auxological and metabolic parameters and all correlations among variables were corrected for age and pubertal status through a logistic regression model. The Pearson's correlation was performed among continuous variables with normal distribution, while the correlations among continuous variables without normal distribution were determined by using the Spearman's test (non-parametric equivalent for Pearson test). To evaluate the independent variables influencing C-peptide levels, a linear regression model was performed. A p value $<0.05$ was considered statistically significant.

\section{Results}

The clinical and biochemical features of control subjects, GHD children at diagnosis and after 12 and 24 months of GHT are shown in Table 1 and Table 2.

\subsection{Clinical and hormonal parameters}

No significant difference in height, BMI and WC between GHD children at baseline and control subjects was found (Table 1). Conversely, as expected, GHD children at baseline showed significantly lower growth velocity SD $(-2.5 \pm 0.6$ vs. $-1.7 \pm 0.6 ; \mathrm{p}<0.001)$, IGF-I SDS $(-1.55 \pm 0.76$ vs. $0.74 \pm 0.39 ; \mathrm{p}<0.001), \mathrm{GH}$ peak after arginine $(3.7 \pm 2.7$ vs. $11.8 \pm 5.6 \mu \mathrm{g} / \mathrm{l} ; \mathrm{p}<0.001)$ and GST $(5.8 \pm 4.9$ vs. $16.5 \pm 8.7 \mu \mathrm{g} / \mathrm{l} ; \mathrm{p}<0.001)$ than controls.

In the GHD group, the growth significantly increased after 12 months of GHT (height SDS: $-1.8 \pm 0.6$ vs. $-2.2 \pm 0.9 ; \mathrm{p}<0.001$; growth velocity SD: $1.6 \pm 0.8$ vs. $-2.5 \pm 0.6 ; p<0.001)$, with a concomitant significant increase in WC SDS $0.01(-1.63-2.22)$ vs. $-0.27(-2.50-$ $1.94) ; \mathrm{p}=0.002]$ and IGF-I SDS $(1.24 \pm 0.84$ vs. $-1.55 \pm 0.76$; $\mathrm{p}<0.001$ ) and with a subsequent stability or a slight and not statistically significant increase, after 24 months (Table 2).

\subsection{Glucose metabolism and insulin sensitivity indexes}

All GHD children at baseline showed a normal glucose tolerance with normal fasting glucose $(4.2 \pm 0.6 \mathrm{mmol} / \mathrm{l})$, insulin [2.8 (0.2-8.7) $\mathrm{IU} / \mathrm{ml}$ ] and HbA1c (5.2 $\pm 0.3 \%$ ) levels, without significant difference of these parameters compared to the control subjects. Similarly, no difference was found in AUC $_{\mathrm{GLU}}$, Homa-IR, QUICKI and ISI Matsuda between GHD children at baseline and control subjects (Table 1 ).

After 12 months of GHT, despite all children remained with a normal glucose tolerance, a significant increase in fasting glucose ( $4.6 \pm 0.5 \mathrm{vs}$. $4.2 \pm 0.6 \mathrm{mmol} / \mathrm{l} ; \mathrm{p}=0.003)$, fasting insulin [8 (0.2-26.7) vs. $2.8(0.2-$ 8.7) $\mathrm{IU} / \mathrm{ml} ; \mathrm{p}<0.001]$ and Homa-IR [(1.7 (0-5.8) vs. 0.53 (0.03-2.69); $\mathrm{p}<0.001$ ], with a significant decrease in QUICKI $(0.37 \pm 0.08$ vs. $0.45 \pm 0.10 ; \mathrm{p}=0.003)$ and ISI Matsuda [(5.7 (2.1-30.9) vs. 14.5 (2.0-34.7); $<<0.001$ ] was found, without significant change in AUC $_{\mathrm{GLU}}$ and Hba1c levels. These trends were maintained after 24 months of treatment, although not statistically significant (Table 2).
Table 1

Clinical and biochemical features of GHD children at diagnosis (baseline) and control subjects. Data are presented as rates and proportions for the categorical data and as mean \pm standard deviation (SD), mean \pm standard deviation score (SDS, when indicated) or median \pm interquartile range (IR) for the continuous variables, when appropriate.

\begin{tabular}{|c|c|c|c|}
\hline & \multirow{2}{*}{$\begin{array}{l}\text { Control group } \\
\text { (N. 56) }\end{array}$} & \multirow{2}{*}{$\frac{\text { GHD at baseline }}{\text { (N. } 48)}$} & \multirow[t]{2}{*}{$\mathrm{p}^{*}$} \\
\hline & & & \\
\hline Gender & & & 235 \\
\hline Males & $39(70 \%)$ & $32(67 \%)$ & \\
\hline Females & $17(30 \%)$ & $16(33 \%)$ & \\
\hline Age (years) & $10.2 \pm 1.3$ & $9.8 \pm 2.1$ & 0.113 \\
\hline Height (SDS) & $-2.1 \pm 0.6$ & $-2.2 \pm 0.9$ & 0.423 \\
\hline $\begin{array}{l}\text { Height in comparison to the } \\
\text { midparental height (SDS) }\end{array}$ & $-0.82 \pm 0.11$ & $-1.90 \pm 0.10$ & $<0.001$ \\
\hline Growth velocity (cm/year) & $4.2 \pm 0.5$ & $3.2 \pm 0.5$ & $<0.001$ \\
\hline Growth velocity (SDS) & $-1.7 \pm 0.6$ & $-2.5 \pm 0.6$ & $<0.001$ \\
\hline BMI $\left(\mathrm{kg} / \mathrm{m}^{2}\right)$ & $\begin{array}{l}16.4 \\
(11.9-26.8)\end{array}$ & $\begin{array}{l}17.2 \\
(12.5-23.1)\end{array}$ & 0.468 \\
\hline BMI (SDS) & $\begin{array}{l}-0.6 \\
(-1.5-2.1)\end{array}$ & $\begin{array}{l}-0.4 \\
(-1.4-1.5)\end{array}$ & 0.463 \\
\hline WC (SDS) & $\begin{array}{l}0.16 \\
(-1.61-1.39)\end{array}$ & $\begin{array}{l}-0.27 \\
(-2.50-1.94)\end{array}$ & 0.085 \\
\hline IGF-I (SDS) & $0.74 \pm 0.39$ & $-1.55 \pm 0.66$ & $<0.001$ \\
\hline $\begin{array}{l}\text { GH peak after arginine } \\
\text { test }(\mu \mathrm{g} / \mathrm{l})\end{array}$ & $\begin{array}{l}11.3 \\
(3.2-22.1)\end{array}$ & $3.5(0.07-7.8)$ & $<0.001$ \\
\hline GH peak after GST $(\mu \mathrm{g} / \mathrm{l})$ & $\begin{array}{l}12.8 \\
(3.9-43.3)\end{array}$ & $5.6(0.12-8.4)$ & $<0.001$ \\
\hline \multicolumn{4}{|l|}{ Glucose metabolism } \\
\hline Fasting glucose $(\mathrm{mmol} / \mathrm{l})$ & $4.2 \pm 0.5$ & $4.2 \pm 0.6$ & 0.847 \\
\hline $\mathrm{AUC}_{\mathrm{GLU}}(\mathrm{mmol} / \mathrm{l})$ & $\begin{array}{l}750 \\
(593-1064)\end{array}$ & $\begin{array}{l}734 \\
(472-1297)\end{array}$ & 0.793 \\
\hline HbA1c (\%) & $5.2 \pm 0.3$ & $5.2 \pm 0.3$ & 0.777 \\
\hline \multicolumn{4}{|l|}{ Insulin secretion indexes } \\
\hline Fasting insulin (IU/ml) & $3.2(0.2-8.8)$ & $2.8(0.2-8.7)$ & 0.743 \\
\hline $\operatorname{AUC}_{\mathrm{INS}}(\mathrm{IU} / \mathrm{ml})$ & $\begin{array}{l}4303 \\
(789-9693)\end{array}$ & $\begin{array}{l}3397 \\
(528-12,973)\end{array}$ & 0.052 \\
\hline Homa- $\beta$ & $\begin{array}{l}12.3 \\
(0.5-38.7)\end{array}$ & $9.7(2.5-49.1)$ & 0.464 \\
\hline Insulinogenic index & $0.7(0-2.6)$ & $0.6(0-3.0)$ & 0.096 \\
\hline Fasting C-peptide (ng/ml) & $1.5 \pm 0.5$ & $1.3 \pm 0.6$ & 0.166 \\
\hline $\operatorname{AUC}_{\text {Cpep }}(\mathrm{ng} / \mathrm{ml})$ & $537 \pm 225$ & $436 \pm 231$ & 0.006 \\
\hline \multicolumn{4}{|l|}{ Insulin sensitivity indexes } \\
\hline Homa-IR & $\begin{array}{l}0.59 \\
(0.03-1.63)\end{array}$ & $\begin{array}{l}0.53 \\
(0.03-2.69)\end{array}$ & 0.814 \\
\hline QUICKI & $0.45 \pm 0.12$ & $0.45 \pm 0.10$ & 0.793 \\
\hline ISI-Matsuda & $\begin{array}{l}12.1 \\
(4.6-38.4)\end{array}$ & $14.5(2.0-34.7)$ & 0.457 \\
\hline
\end{tabular}

AUC: area under the curve; OGTT: oral glucose tolerance test; GST: glucagon stimulation test.

AUC $_{\text {INS }}$ : AUC of insulin during OGTT; AUC ${ }_{\text {Cpep }}$ : AUC of C-peptide during GST. $\mathrm{p}^{*}$ value corrected for age and pubertal status.

\subsection{Insulin secretion indexes}

At baseline, no significant difference was found in Homa- $\beta$, insulinogenic index and $\mathrm{AUC}_{\mathrm{INS}}$ between GHD children and control subjects, while GHD children showed significant lower AUC $_{\text {cpep }}$ during GST ( $436 \pm 231$ vs. $537 \pm 225 \mathrm{ng} / \mathrm{ml} ; \mathrm{p}=0.006)$ and lower fasting C-peptide, although non statistically significant (Table 1 ). A significant positive correlation of both fasting C-peptide and AUC $_{\text {Cpep }}$ with IGF-I SDS ( $p=0.007$ and $p<0.001$, respectively), height SDS (both $\mathrm{p}<0.001$ ), BMI SDS (both $\mathrm{p}<0.001$ ) and WC SDS (both $\mathrm{p}<0.001$ ) was found at baseline. Similarly, the other indexes of insulin secretion (fasting insulin, $A U C_{\text {INS }}$, Homa- $\beta$ ), with the exception of insulinogenic index, were significantly correlated with the clinical and hormonal parameters (Table 3). At multivariate analysis, both fasting C-peptide (beta 0.307 , $\mathrm{p}=0.016$ ) and $\mathrm{AUC}_{\mathrm{Cpep}}$ (beta $0.379, \mathrm{p}=0.002$ ) independently 
Table 2

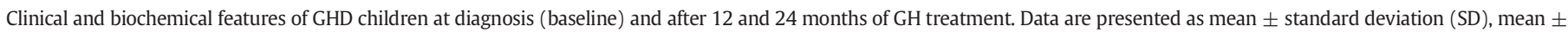
standard deviation score (SDS, when indicated) or median \pm interquartile range (IR) for the continuous variables, when appropriate.

\begin{tabular}{|c|c|c|c|c|c|}
\hline & Baseline & 12 months & 24 months & $\mathrm{p}$ & $\mathrm{p}^{*}$ \\
\hline Height (SDS) & $-2.2 \pm 0.9$ & $-1.8 \pm 0.6$ & $-1.3 \pm 0.6$ & $<0.001$ & 0.077 \\
\hline Growth velocity (cm/year) & $3.2 \pm 0.5$ & $8.5 \pm 2.4$ & $7.9 \pm 2.1$ & $<0.001$ & 0.572 \\
\hline Growth velocity (SDS) & $-2.5 \pm 0.6$ & $1.6 \pm 0.8$ & $1.4 \pm 0.3$ & $<0.001$ & 0.665 \\
\hline BMI $\left(\mathrm{kg} / \mathrm{m}^{2}\right)$ & $17.2(12.5-23.1)$ & $17(12.7-27.2)$ & $18(14.3-24)$ & 0.081 & 0.051 \\
\hline BMI (SDS) & $-0.4(-1.4-1.5)$ & $-0.3(-1.3-1.8)$ & $0.01(-1.3-1.3)$ & 0.106 & 0.058 \\
\hline WC (SDS) & $-0.27(-2.50-1.94)$ & $0.01(-1.63-2.22)$ & $0.05(-1.14-2.06)$ & 0.002 & 0.859 \\
\hline IGF-I (SDS) & $-1.55 \pm 0.66$ & $1.14 \pm 0.44$ & $1.29 \pm 0.31$ & $<0.001$ & 0.113 \\
\hline \multicolumn{6}{|l|}{ Glucose metabolism } \\
\hline Fasting glucose (mmol/l) & $4.2 \pm 0.6$ & $4.6 \pm 0.5$ & $4.7 \pm 0.5$ & 0.003 & 0.883 \\
\hline $\mathrm{AUC}_{\mathrm{GLU}}(\mathrm{mmol} / \mathrm{l})$ & $734(472-1297)$ & $745(531-1261)$ & $679(526-858)$ & 0.414 & 0.929 \\
\hline HbA1c (\%) & $5.2 \pm 0.3$ & $5.3 \pm 3.1$ & $5.2 \pm 0.4$ & 0.053 & 0.907 \\
\hline \multicolumn{6}{|l|}{ Insulin secretion indexes } \\
\hline Fasting insulin (IU/ml) & $2.8(0.2-8.7)$ & $8(0.2-26.7)$ & $9.5(0.3-25.3)$ & $<0.001$ & 1 \\
\hline $\mathrm{AUC}_{\mathrm{INS}}(\mathrm{IU} / \mathrm{ml})$ & $3397(528-12,973)$ & $5043(592-20,506)$ & $5386(2224-10,477)$ & 0.213 & 0.959 \\
\hline Homa- $\beta$ & $9.7(2.5-49.1)$ & $28.8(1.6-76.6)$ & $35.2(2.2-76.7)$ & $<0.001$ & 0.496 \\
\hline Insulinogenic Index & $0.7(0-2.6)$ & $0.9(0.1-4.0)$ & $1.4(0.3-4.0)$ & 0.112 & 0.878 \\
\hline Fasting C-peptide (ng/ml) & $1.3 \pm 0.6$ & $1.7 \pm 0.6$ & $1.8 \pm 0.6$ & 0.002 & 0.457 \\
\hline $\operatorname{AUC}_{\text {Cpep }}(\mathrm{ng} / \mathrm{ml})$ & $436 \pm 231$ & $521 \pm 211$ & $604 \pm 213$ & $<0.001$ & 0.067 \\
\hline \multicolumn{6}{|l|}{ Insulin sensitivity indexes } \\
\hline Homa-IR & $0.53(0.03-2.69)$ & $1.7(0-5.8)$ & $2.2(0-6.3)$ & $<0.001$ & 1 \\
\hline QUICKI & $0.45 \pm 0.10$ & $0.37 \pm 0.08$ & $0.36 \pm 0.08$ & 0.003 & 0.621 \\
\hline ISI-Matsuda & $14.5(2.0-34.7)$ & $5.7(2.1-30.9)$ & $5.1(2.7-39.9)$ & $<0.001$ & 0.959 \\
\hline
\end{tabular}

AUC: area under the curve; OGTT: oral glucose tolerance test; GST: glucagon stimulation test.

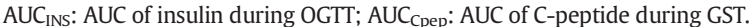

$\mathrm{p}=$ difference between baseline and 12 months.

$\mathrm{p}^{*}=$ difference between 12 and 24 months.

correlated with IGF-I SDS, while no significant correlation was found for all other insulin secretion indexes (Table 4; Fig. 1A and B).

After 12 months of therapy a significant increase in Homa- $\beta$ [28.8 (1.6-76.6) vs. 9.7 (2.5-49.1); $<<0.001$ ], fasting C-peptide $(1.7 \pm 0.6$ vs. $1.3 \pm 0.6 \mathrm{ng} / \mathrm{ml} ; \mathrm{p}=0.002)$ and AUC $_{\text {Cpep }}$ during GST $(521 \pm 211$ vs. $436 \pm 231 \mathrm{ng} / \mathrm{ml} ; p<0.001$ ) was found, without significant change in insulinogenic index and $\mathrm{AUC}_{\mathrm{INS}}$. These trends were maintained after 24 months of treatment, although not statistically significant (Table 2).

Table 3

Correlation (univariate analysis) among the insulin-secretion indexes and the clinical and hormonal parameters of GHD children at baseline and after 12 months of GH treatment. Data are considered as standard deviation score (SDS) values.

\begin{tabular}{|c|c|c|c|c|}
\hline \multirow[t]{3}{*}{ Independent variables } & \multicolumn{4}{|c|}{ Dependent variable: Fasting C-peptide } \\
\hline & \multicolumn{2}{|c|}{ Baseline } & \multicolumn{2}{|c|}{12 months } \\
\hline & $\mathrm{r}$ & $\mathrm{p}$ & $\mathrm{r}$ & $\mathrm{p}$ \\
\hline Height (SDS) & 0.374 & $<0.001$ & 0.418 & 0.030 \\
\hline BMI (SDS) & 0.484 & $<0.001$ & 0.485 & $<0.001$ \\
\hline WC (SDS) & 0.391 & $<0.001$ & 0.606 & $<0.001$ \\
\hline IGF-I (SDS) & 0.251 & 0.007 & 0.778 & $<0.001$ \\
\hline \multicolumn{5}{|l|}{ AUCCрер } \\
\hline Height (SDS) & 0.476 & $<0.001$ & 0.423 & 0.028 \\
\hline BMI (SDS) & 0.593 & $<0.001$ & 0.467 & 0.001 \\
\hline WC (SDS) & 0.458 & $<0.001$ & 0.565 & $<0.001$ \\
\hline IGF-I (SDS) & 0.393 & $<0.001$ & 0.862 & $<0.001$ \\
\hline \multicolumn{5}{|l|}{ Fasting insulin } \\
\hline Height (SDS) & 0.325 & 0.001 & 0.128 & 0.534 \\
\hline BMI (SDS) & 0.519 & $<0.001$ & 0.336 & 0.016 \\
\hline WC (SDS) & 0.551 & $<0.001$ & 0.361 & 0.007 \\
\hline IGF-I (SDS) & 0.216 & 0.027 & 0.420 & 0.004 \\
\hline \multicolumn{5}{|l|}{ AUCINS } \\
\hline Height (SDS) & 0.239 & 0.014 & 0.259 & 0.257 \\
\hline BMI (SDS) & 0.509 & $<0.001$ & 0.375 & 0.017 \\
\hline WC (SDS) & 0.531 & $<0.001$ & 0.336 & 0.055 \\
\hline IGF-I (SDS) & 0.232 & 0.019 & 0.151 & 0.394 \\
\hline
\end{tabular}

Table 4

Independent variables influencing the insulin secretion indexes at multivariate analysis (multiple linear regression). Data are considered as standard deviation score (SDS) values.

\begin{tabular}{|c|c|c|c|c|c|c|}
\hline \multirow[t]{3}{*}{ Independent variables } & \multicolumn{6}{|c|}{ Dependent variable: Fasting C-peptide } \\
\hline & \multirow{2}{*}{$\frac{\text { Baseline }}{\mathrm{B}}$} & \multirow{2}{*}{$\frac{12 \text { months }}{\mathrm{SE}}$} & \multirow[b]{2}{*}{$\mathrm{p}$} & \multirow[b]{2}{*}{ B } & \multirow[b]{2}{*}{ SE } & \multirow[b]{2}{*}{$\mathrm{p}$} \\
\hline & & & & & & \\
\hline Height (SDS) & 0.017 & 0.064 & 0.887 & 0.121 & 0.130 & 481 \\
\hline BMI (SDS) & 0.445 & 0.039 & 0.070 & -0.287 & 0.051 & 0.349 \\
\hline WC (SDS) & -0.034 & 0.109 & 0.109 & 0.215 & 0.990 & 0.566 \\
\hline IGF-I (SDS) & 0.307 & 0.001 & 0.016 & 0.783 & 0.001 & 0.001 \\
\hline \multicolumn{7}{|l|}{ AUC $_{\text {Cpep }}$} \\
\hline Height (SDS) & 0.201 & 2.880 & 0.072 & 0.074 & 2.906 & 0.467 \\
\hline BMI (SDS) & 0.434 & 1.741 & 0.058 & -0.088 & 1.113 & 0.625 \\
\hline WC (SDS) & -0.596 & 5.150 & 0.252 & 0.348 & 7.608 & 0.300 \\
\hline IGF-I (SDS) & 0.379 & 0.293 & 0.002 & 0.880 & 0.127 & $<0.001$ \\
\hline \multicolumn{7}{|l|}{ Fasting insulin } \\
\hline Height (SDS) & 0.139 & 0.370 & 0.199 & - & - & - \\
\hline BMI (SDS) & 0.111 & 0.230 & 0.614 & -0.317 & 0.707 & 0.441 \\
\hline WC (SDS) & 0.299 & 2.188 & 0.304 & 0.433 & 2.410 & 0.247 \\
\hline IGF-I (SDS) & 0.081 & 0.004 & 0.477 & 0.320 & 0.007 & 0.130 \\
\hline \multicolumn{7}{|l|}{$\mathrm{AUC}_{\mathrm{INS}}$} \\
\hline Height (SDS) & -0.007 & 3.634 & 0.956 & - & - & - \\
\hline BMI (SDS) & 0.272 & 1.770 & 0.291 & 0.375 & 1.500 & 0.117 \\
\hline WC (SDS) & 0.088 & 0.415 & 0.680 & - & - & - \\
\hline IGF-I (SDS) & 0.104 & 2.954 & 0.436 & - & - & - \\
\hline \multicolumn{7}{|l|}{ Homa- $\beta$} \\
\hline Height (SDS) & 0.166 & 1.610 & 0.114 & - & - & - \\
\hline BMI (SDS) & 0.081 & 0.999 & 0.704 & 0.114 & 1.094 & 0.522 \\
\hline WC (SDS) & 0.260 & 2.971 & 0.205 & - & - & - \\
\hline IGF-I (SDS) & 0.083 & 0.016 & 0.448 & 0.346 & 0.025 & 0.058 \\
\hline \multicolumn{7}{|l|}{ Insulinogenic index } \\
\hline Height (SDS) & - & - & - & - & - & - \\
\hline BMI (SDS) & - & - & - & - & - & - \\
\hline WC (SDS) & - & - & - & 0.449 & 0.156 & 0.105 \\
\hline IGF-I (SDS) & - & - & - & - & - & - \\
\hline
\end{tabular}


A

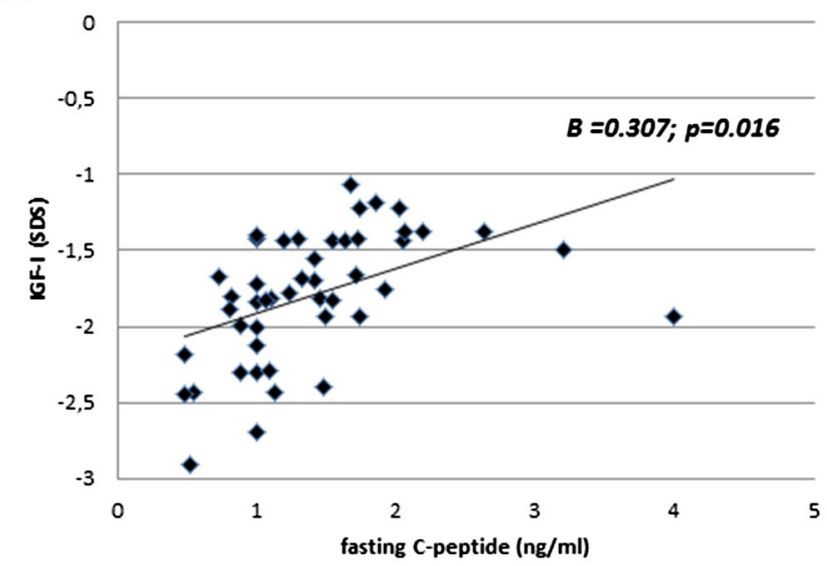

C

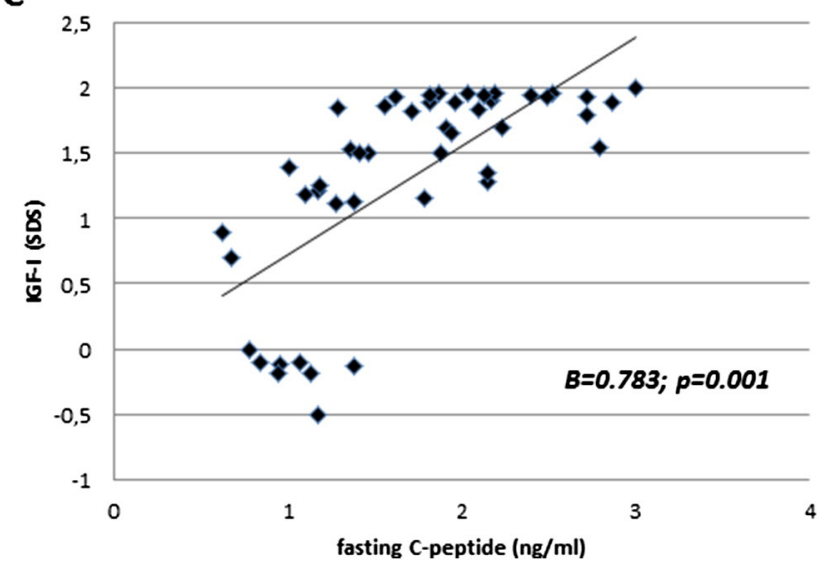

B

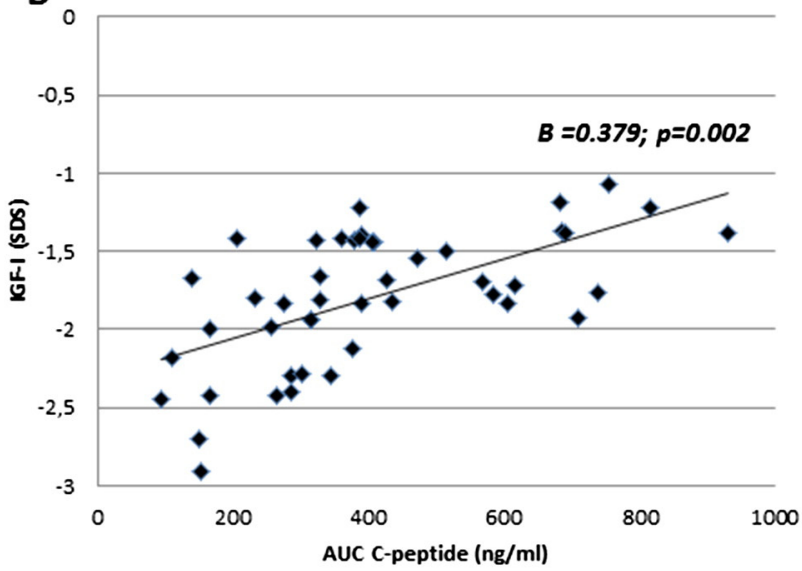

D

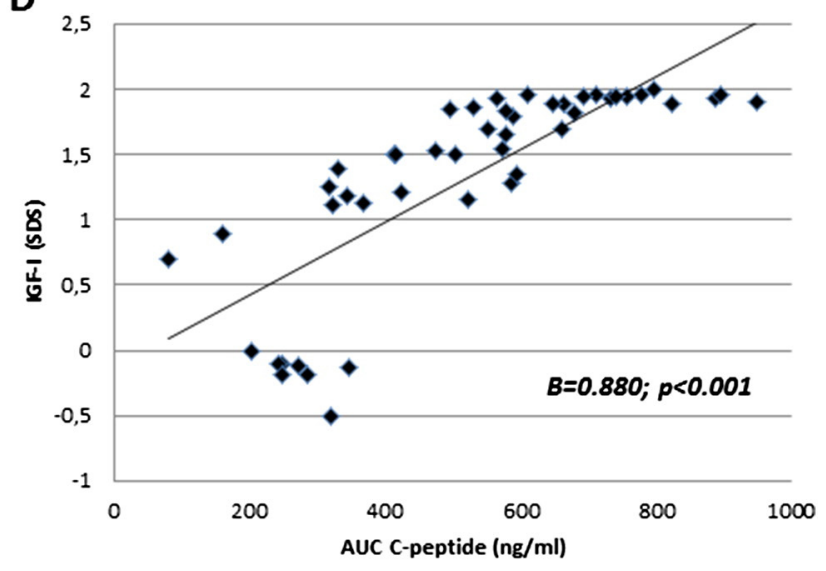

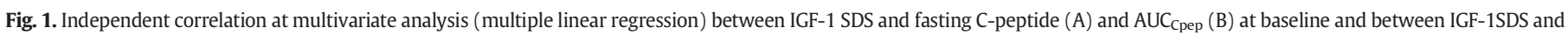
fasting C-peptide (C) and $\mathrm{AUC}_{\mathrm{Cpep}}(\mathrm{D})$ after 12 months of GH therapy.

After 12 months of treatment a significant positive correlation of both fasting $C$-peptide and AUC $_{\text {Cpep }}$ with IGF-I SDS (both $\mathrm{p}<0.001$ ), height SDS ( $p=0.030$ and $p=0.028$, respectively), BMI SDS $(\mathrm{p}<0.001$ and $\mathrm{p}=0.001$, respectively) and WC SDS (both $\mathrm{p}<0.001$ ) was found. We found that fasting insulin significantly correlated with IGF-I SDS ( $p=0.004)$, BMI SDS $(p=0.016)$ and WC SDS $(p=0.007)$, Homa- $\beta$ correlated with IGF-I SDS $(p=0.005)$ and BMI SDS $(p=$ $0.030), A C_{\text {INS }}$ correlated with BMI SDS $(\mathrm{p}=0.017)$ and insulinogenic index correlated with WC SDS ( $\mathrm{p}=0.001$ ) (Table 3). At multivariate analysis, both fasting C-peptide (beta 0.783, $\mathrm{p}=0.001$ ) and $\mathrm{AUC}_{\mathrm{Cpep}}$ (beta $0.880, \mathrm{p}<0.001$ ) independently correlated with IGF-I SDS, while no significant correlation was found for the other insulin secretion indexes (Table 4; Fig. 1C and D).). These data have not been confirmed at 24 months of treatment (data not shown).

As additional analysis, we grouped all GHD children according to gender and family history of diabetes and we did not found any difference in all metabolic parameters between males and females and between children with or without family history of diabetes (data not shown).

\section{Discussion}

The mutual relationship between insulin-secretion and GH/IGF-I axis is well known in healthy subjects, as well as in diabetic patients $[16,17]$. Given the insulin antagonist action of $\mathrm{GH}$, in addition to the direct stimulatory effect on $\beta$-cell, GHT can lead to glucose metabolism impairment through decreased insulin sensitivity and impaired pancreatic $\beta$-cell function [12]. Therefore, a reliable evaluation of the insulin secretion in GHD children during GHT is very important. In this prospective study we demonstrated the greater reliability of C-peptide than insulin levels as an estimate of insulin secretion in children with GHD, both at baseline and during the first 12 months of GHT. Indeed, we showed the limitation of the insulin-derived indexes of insulin secretion because of their lack of correlation with the parameters used in the clinical practice to monitor GHT. The effect of GHT on glucose metabolism is predominantly insulin antagonistic. A marked hyperinsulinemia and a resistance to the action of insulin, as demonstrated by euglycemic clamp studies in healthy subjects, have been demonstrated [7,32]. We already demonstrated an increase in HOMA-IR, related to the increased insulin levels and without any untoward effect on glucose metabolism, in a small group of GHD children after GHT [33]. A subtle form of insulin resistance, defined as a diminished biological response to a given insulin concentration, can be assumed as the mechanism by which basal glucose turnover did not change after GHT despite the increase in insulin levels. Indeed, if the sensitivity to insulin was maintained, a reduction in glucose levels should occur. We showed a significant increase in fasting glucose levels despite the concomitant increase in insulin during GHT, suggesting the inability of the $\beta$-cells to adequately compensate the insulin resistance state. However, in this study, only fasting glucose showed a significant increase, although it remained within the limits of normality, while no significant change was found in $\mathrm{AUC}_{\mathrm{GLU}}$ and $\mathrm{HbA1C}$ levels, and these data could explain the maintenance of normal glucose tolerance during GHT. Similarly, in adult GHD patients GHT seems to result in a significant increase in both insulin and C-peptide levels, but insufficient to match the change in insulin sensitivity, as demonstrated by the trend to increase in stimulated glucose levels [19,34]. 
We already demonstrated in GHD children after GHT a decrease in insulin sensitivity, evaluated using the gold standard euglycemic hyperinsulinemic clamp method, and a concomitant inadequate $\beta$-cell compensation to the decreased insulin sensitivity [6], but a limit of these evaluations was the use of insulin secretion indexes derived from insulin levels [35-37].

It is well known that $\beta$-cell function cannot be accurately estimated on the basis of the endogenous insulin levels because of the pulsatile release pattern and the short half-life and a more accurate estimation could be provided by C-peptide, a byproduct of insulin production with a half-life of $30 \mathrm{~min}$. Since insulin and C-peptide are cosecreted by $\beta$-cells on an equimolar basis, the stimulated secretion of $C$-peptide is widely accepted as a measure of $\beta$-cell function [38-40]. Some authors have also demonstrated a stronger correlation among OGTT and the gold standard insulin secretion method (hyperglycemic clamp) for C-peptide than for insulin, probably related to the difference in C-peptide kinetics and insulin clearance $[41,42]$. For these reasons, the use of the insulin concentration as an index of insulin secretion during GHT might be misleading, although very few studies evaluated the Cpeptide levels in GHD children. Using the hyperglycemic clamp Heptulla et al. have demonstrated a significant increase in both the first and second phase insulin and C-peptide response in short children after GHT [18]. In our study, the consistent elevation in plasma insulin (and consequently in Homa- $\beta$ index) and C-peptide levels in the fasting state and their responses to the stimulation by OGTT or glucagon indicates that insulin synthesis and secretion were increased by GHT, which may be due to the insulin sensitivity reduction, as demonstrated by the increase in Homa-IR and the decrease in QUICKI and ISI Matsuda, as well as to the direct insulinotropic effect of GH on the $\beta$-cells. Indeed, in vitro studies of rat pancreatic cells have demonstrated that $\mathrm{GH}$ directly acts on $\beta$-cells to stimulate both expression of the insulin gene and DNA synthesis and cell replication independently of the plasma glucose concentration [9, 10]. Our findings are supported by the data of Walker et al. who has shown a consistent increase both in insulin and C-peptide concentrations in prepubertal children after 12 months of GHT. However, these authors did not evaluate any correlation among clinical, hormonal and metabolic parameters [22].

In the current study we found that, among all indexes of insulin secretion evaluated, only C-peptide levels, especially those stimulated after GST, correlate with IGF-I both after 12 months of GHT and also at baseline. Indeed, in untreated GHD with lower IGF-I concentrations we found lower C-peptide levels compared with control subjects, whereas insulin levels and the other indexes of insulin secretion were similar. A decrease in hepatic clearance of insulin during GHT can be hypothesized in determining a greater peripheral hyperinsulinemia. Therefore, measuring C-peptide may override problems associated with the difference in insulin clearance and its effect on circulating insulin levels and it may be more informative than insulin levels in the evaluation of the insulin secretion [21]. In addition, the strong correlation between C-peptide and IGF-I levels leads us to consider C-peptide a very reliable marker during GHT. These findings strongly support that insulin secretion is a major co-regulator of IGF-I levels also in GHD children and they are consistent with other studies that have reported a correlation between C-peptide and IGF-I levels [16,17].

A limit of this study is represented by the lack of data of the control group during the follow-up, mainly due to ethical reasons in submitting these healthy children to GST once a year. Indeed, we cannot rule out with certainty that these results are due to some other factor, in addition to the GHT. Even if all children were prepubertal, a minimal role played by the change of gonadal hormones during the follow-up cannot be ruled out, although nobody changed its pubertal stage at visual inspection, no difference was found between males and females and although the statistical differences in auxological and metabolic parameters were corrected for age and pubertal status.

In conclusion, a relationship between insulin-secretion and IGF-I levels is documented in GHD children both at baseline and during
GHT and C-peptide has proven to be the most reliable indicator of insulin secretion correlated to IGF-I levels. Therefore, we suggest the use of GST not only as a diagnostic test for the GH assessment at baseline but also as a useful tool, instead of OGTT, for the evaluation of insulin secretion during GHT in children, at least during the first year, to simplify the test in clinical practice and to reduce costs and resources. Additional larger case-control studies with longer follow-up will validate these results.

\section{Disclosure statement}

The authors have nothing to disclose.

\section{Grants}

This research did not receive any specific grant from any funding agency in the public, commercial or non-profit sector.

\section{Conflict of interest}

All authors declare that there is no conflict of interest that could be perceived as prejudicing the impartiality of the research reported.

\section{References}

[1] A. Vijayakumar, R. Novosyadlyy, Y. Wu, et al., Biological effects of growth hormone on carbohydrate and lipid metabolism, Growth Hormon. IGF Res. 20 (2010) 1-7.

[2] A.M. Boot, M.A. Engels, G.J. Boerma, et al., Changes in bone mineral density, body composition, and lipid metabolism during growth hormone $(\mathrm{GH})$ treatment in children with GH deficiency, J. Clin. Endocrinol. Metab. 82 (1997) 2423-2428.

[3] D. Capalbo, G. Mattace Raso, A. Esposito, et al., Cluster of cardiometabolic risk factors in children with GH deficiency: a prospective, case-control study, Clin. Endocrinol. 80 (2013) 856-862.

[4] D. Capalbo, A. Esposito, R. Di Mase, et al., Update on early cardiovascular and metabolic risk factors in children and adolescents affected with growth hormone deficiency, Minerva Endocrinol. 37 (2012) 379-389.

[5] J. Cordoba-Chacon, M.D. Gahete, O.P. McGuinness, et al., Differential impact of selective $\mathrm{GH}$ deficiency and endogenous $\mathrm{GH}$ excess on insulinmediated actions in muscle and liver of male mice, Am. J. Physiol. Endocrinol. Metab. 307 (2014) E928-E934.

[6] A. Ciresi, M.C. Amato, C. Giordano, Reduction in insulin sensitivity and inadequate $\beta$ cell capacity to counteract the increase in insulin resistance in children with idiopathic growth hormone deficiency during 12 months of growth hormone treatment, J. Endocrinol. Investig. 38 (2015) 351-359.

[7] P.R. Bratusch-Marrain, D. Smith, R.A. DeFronzo, The effect of growth hormone on glucose metabolism and insulin secretion in man, J. Clin. Endocrinol. Metab. 55 (1982) 973-982.

[8] R.A. Rizza, J.E. Gerich, Physiological concentrations of growth hormone exert insulin-like and insulin antagonistic effects on both hepatic and extrahepatic tissues in man, J. Clin. Endocrinol. Metab. 53 (1981) 556-559.

[9] A. Rabinovitch, C. Quigley, M.M. Rechler, Growth hormone stimulates islet B-cell replication in neonatal rat pancreatic monolayer cultures, Diabetes 32 (1983) 307-312.

[10] B. Formby, A. Ullrich, L. Coussens, et al., Growth hormone stimulates insulin gene expression in cultured human fetal pancreatic islets, J. Clin. Endocrinol. Metab. 66 (1988) 1075-1079.

[11] F. Zhang, A. Sjöholm, Q. Zhang, Growth hormone signaling in pancreatic beta-cellscalcium handling regulated by growth hormone, Mol. Cell. Endocrinol. 297 (2009) 50-57.

[12] N. Møller, P.C. Butler, M.A. Antsiferov, et al., Effects of growth hormone on insulin sensitivity and forearm metabolism in normal man, Diabetologia 32 (1989) 105-110.

[13] P. Saenger, Metabolic consequences of growth hormone treatment in paediatric practice, Horm. Res. 53 (2000) 60-69.

[14] C.J. Child, A.G. Zimmermann, R.S. Scott, et al., GeNeSIS international advisory board, prevalence and incidence of diabetes mellitus in GH-treated children and adolescents: analysis from the GeNeSIS observational research program, J. Clin. Endocrinol. Metab. 96 (2011) E1025-E1034.

[15] W.S. Cutfield, P. Wilton, H. Bennmarker, et al., Incidence of diabetes mellitus and impaired glucose tolerance in children and adolescents receiving growth-hormone treatment, Lancet 355 (2000) 610-613.

[16] J.S. Sorensen, N.H. Birkebaek, M. Bjerre, et al., Residual $\beta$-cell function and the insulin-like growth factor system in Danish children and adolescents with type 1 diabetes, J. Clin. Endocrinol. Metab. 100 (2015) 1053-1056.

[17] C. Bizzarri, D. Benevento, G. Giannone, et al., Sexual dimorphism in growth and insulin-like growth factor-I in children with type 1 diabetes mellitus, Growth Hormon. IGF Res. 24 (2014) 256-259.

[18] R.A. Heptulla, S.D. Boulware, S. Caprio, et al., Decreased insulin sensitivity and compensatory hyperinsulinemia after hormone treatment in children with short stature, J. Clin. Endocrinol. Metab. 82 (1997) 3234-3238. 
[19] A.M. Rosenfalck, S. Fisker, J. Hilsted, et al., The effect of the deterioration of insulin sensitivity on beta-cell function in growth-hormone-deficient adults following 4 month growth hormone replacement therapy, Growth Hormon. IGF Res. 9 (1999) 96-105.

[20] R.B. Jensen, A. Thankamony, S.M. O'Connell, et al., Baseline IGF-I levels determine insulin secretion and insulin sensitivity during the first year on growth hormone therapy in children born small for gestational age. Results from a North European Multicentre Study (NESGAS), Horm. Res. Paediatr. 80 (2013) 38-46.

[21] F. Bacha, N. Gungor, S.A. Arslanian, Measures of beta-cell function during the oral glucose tolerance test, liquid mixed-meal test, and hyperglycemic clamp test, J. Pediatr. 152 (2008) 618-621.

[22] J. Walker, J.L. Chaussain, P.F. Bougnères, Growth hormone treatment of children with short stature increases insulin secretion but does not impair glucose disposal, J. Clin. Endocrinol. Metab. 69 (1989) 253-258.

[23] W.A. Marshall, J.M. Tanner, Variations in pattern of pubertal changes in girls, Arch Dis. Child. 44 (1969) (291-230).

[24] Growth Hormone Research Society, Consensus guidelines for the diagnosis and treatment of growth hormone $(\mathrm{GH})$ deficiency in childhood and adolescence: summary statement of the GH Research Society. GH Research Society, J. Clin. Endocrinol. Metab. 85 (2000) 3990-3993.

[25] W. Greulich, S. Pyle, Radiographic Atlas of Skeletal Development of the Hand and Wrist, second ed. Stanford University Press, Stanford, CA, 1959.

[26] K.S. Leong, A.B. Walker, I. Martin, et al., An audit of 500 subcutaneous glucagon stimulation tests to assess growth hormone and ACTH secretion in patients with hypothalamic-pituitary disease, Clin. Endocrinol. 54 (2001) 463-468.

[27] S.M. Orme, A. Price, A.P. Weetman, et al., Comparison of the diagnostic utility of the simplified and standard i.m. glucagon stimulation test (IMGST), Clin. Endocrinol. 49 (1998) 773-778.

[28] D.R. Matthews, J.P. Hosker, A.S. Rudenski, et al., Homeostasis model assessment: insulin resistance and beta-cell function from fasting plasma glucose and insulin concentrations in man, Diabetologia 28 (1985) 412-419.

[29] M. Stumvoll, A. Mitrakou, W. Pimenta, et al., Use of the oral glucose tolerance test to assess insulin release and insulin sensitivity, Diabetes Care 23 (2000) 295-301.

[30] A. Katz, S.S. Nambi, K. Mather, et al., Quantitative insulin sensitivity check index: a simple, accurate method for assessing insulin sensitivity in humans, J. Clin. Endocrinol. Metab. 85 (2000) 2402-2410.
[31] M. Matsuda, R.A. DeFronzo, Insulin sensitivity indices obtained from oral glucose tolerance testing: comparison with the euglycemic insulin clamp, Diabetes Care 22 (1999) 1462-1470.

[32] R.G. Rosenfeld, D.M. Wilson, L.A. Dollar, et al., Both human pituitary growth hormone and recombinant DNA-derived human growth hormone cause insulin resistance at a postreceptor site, J. Clin. Endocrinol. Metab. 54 (1982) 1033-1038.

[33] A. Ciresi, M.C. Amato, A. Criscimanna, et al., Metabolic parameters and adipokine profile during GH replacement therapy in children with GH deficiency, Eur. J. Endocrinol. 156 (2007) 353-360.

[34] A.M. Rosenfalck, S. Maghsoudi, S. Fisker, et al., The effect of 30 months of low-dose replacement therapy with recombinant human growth hormone (rhGH) on insulin and C-peptide kinetics, insulin secretion, insulin sensitivity, glucose effectiveness, and body composition in GH-deficient adults, J. Clin. Endocrinol. Metab. 85 (2000) 4173-4181.

[35] R.L. Hanson, R.E. Pratley, C. Bogardus, et al., Evaluation of simple indices of insulin sensitivity and insulin secretion for use in epidemiologic studies, Am. J. Epidemiol. 151 (2000) 190-198.

[36] G. Guzzaloni, G. Grugni, G. Mazzilli, et al., Comparison between beta-cell function and insulin resistance indexes in prepubertal and pubertal obese children, Metabolism 51 (2002) 1011-1016.

[37] L.S. Conwell, S.G. Trost, W.J. Brown, et al., Batch JA. Indexes of insulin resistance and secretion in obese children and adolescents: a validation study, Diabetes Care 27 (2004) 314-319.

[38] A.H. Rubenstein, M.B. Block, J. Starr, F. Melani, D.F. Steiner, Proinsulin and C-peptide in blood, Diabetes 21 (1972) 661-672.

[39] J.P. Palmer, G.A. Fleming, C.J. Greenbaum, et al., C-peptide is the appropriate outcome measure for type 1 diabetes clinical trials to preserve beta-cell function: report of an ADA workshop, 21-22 October 2001, Diabetes 53 (2004) 250-264.

[40] C. Hendriksen, O.K. Faber, J. Drejer, et al., Prevalence of residual B-cell function in insulin-treated diabetics evaluated by the plasma C-peptide response to intravenous glucagon, Diabetologia 13 (1977) 615-619.

[41] K.S. Polonsky, J. Licinio-Paixao, B.D. Given, et al., Use of biosynthetic human C-peptide in the measurement of insulin secretion rates in normal volunteers and type I diabetic patients, J. Clin. Invest. 77 (1986) 98-105.

[42] O.K. Faber, C. Hagen, C. Binder, et al., Kinetics of human connecting peptide in normal and diabetic subjects, J. Clin. Invest. 62 (1978) 197-203. 